\title{
Dapagliflozin Alleviates Myocardial Inflammation by Regulating the Macrophage Polarization and Stat3- related Pathways in Coxsackie Virus B3-induced Acute Viral Myocarditis
}

\author{
Pengcheng Yan \\ Shandong Provincial Hospital \\ Xiaoning Song \\ Shandong Provincial Hospital \\ Joanne Tran \\ University of Portland \\ Runfa Zhou \\ Shandong Provincial Hospital \\ Xinran Cao \\ Shandong Provincial Hospital \\ Gang Zhao (D zg000507@163.com ) \\ Shandong Provincial Hospital https://orcid.org/0000-0002-9939-9001 \\ Haitao Yuan \\ Shandong Provincial Hospital
}

\section{Research Article}

Keywords: Dapagliflozin, Macrophage polarization, Viral myocarditis, Stat3

Posted Date: January 13th, 2022

DOI: https://doi.org/10.21203/rs.3.rs-1200173/v1

License: (c) (i) This work is licensed under a Creative Commons Attribution 4.0 International License. Read Full License 


\section{Abstract}

Viral myocarditis (VMC), which is most prevalently caused by Coxsackievirus B3 (CVB3) infection, is a serious clinical condition characterized by cardiac inflammation. Dapagliflozin, a kind of sodium glucose co-transporters 2(SGLT-2) inhibitor, exhibited protective effects on plenty of inflammatory diseases, while its effect on viral myocarditis has not been studied. Recently we found the protective effect of dapagliflozin on VMC. After CVB3 infection, dapagliflozin were given orally to Balb/c male mice for 8 days and then the severity of myocarditis was assessed. Our results indicated that dapagliflozin significantly alleviated the severity of viral myocarditis, elevated the survival rate, and ameliorated cardiac function. Besides, dapagliflozin can decrease the level of proinflammatory cytokines included IL-1 $\beta$, IL-6, TNF-a. Furthermore, dapagliflozin can inhibit macrophages differentiate to classically activated macrophages (M1) in cardiac tissue and activate the Stat3 signal pathway which is reported to promote polarization of the alternatively activated macrophage (M2). In conclusion, our study demonstrates that dapagliflozin alleviates myocardial inflammation by regulating the macrophage polarization and Stat3related pathways in coxsackie virus B3-induced acute viral myocarditis.

\section{Introduction}

Myocarditis can be caused by a variety of pathogens included virus, bacteria, fungi, Chlamydia, rickettsia, protozoa, toxins and etc. Viral myocarditis (VMC) is mainly caused by enterovirus, especially coxsackievirus group B3 (CVB3). In recent years, the incidence of VMC is increasing steadily in the whole word, although most viral myocarditis is self-limited, some acute myocarditis may develop into chronic stage, finally generating dilated cardiomyopathy (DCM) and congestive heart failure $[1,2]$. The pathogenesis of VMC induced by CVB3 includes direct viral injury and indirect injury caused by immune response. After CVB3 infection, a numerous of inflammatory factors are produced such as IFN- $y$, IL-1 $\beta$ and TNF- $a$, which will lead to the migration and infiltration of macrophage, and eventually lead to autoimmune injury[3, 4].

There is a remarkable gender difference in the type of cardiac macrophages in VMC. The macrophages infiltrated in the heart of female mice are mainly M2-type, which secretes anti-inflammatory factors such as IL-10. While in male mouse are mainly M1-type, which secretes pro-inflammatory factors such as IL-6, IL-12 and chemokine MCP-1, etc. [5, 6]. Thus, inhibiting macrophage polarizing to M1-type or promoting macrophage polarizing to M2-type can alleviate the myocardial inflammation in male mice[7, 8].

Dapagliflozin, a kind of sodium glucose co-transporters 2(SGLT-2) inhibitor, targets against renal glucose reabsorption. Recently, several clinical trials showed the cardiovascular protective effects of SGLT-2 inhibitors[9]. Both DAPA-HF and EMPEROR-Reduced trials showed that in patients with heart failure, SGLT2 inhibitor reduced the combined risk of cardiovascular death or hospitalization for heart failure[10, 11], and consistent with the above clinical trials, several studies indicated that the benefits of SGLT-2 inhibitors depending on its anti-inflammatory effects[12-14]. SGLT-2 inhibitors can reduce inflammatory markers such as interleukin-6 (IL-6), monocyte chemoattractant protein-1 (MCP-1) and tumor necrosis 
factor (TNF-a), thereby alleviating arteriosclerosis in diabetes animal models[15]. Terami et al. treated diabetic mice with dapagliflozin and found that macrophage infiltration in the treatment group and the expressions of inflammatory genes such as MCP-1 and transforming growth factor- $\beta$ (TGF- $\beta$ ) all decreased[16]. Weiling Leng et al. found that dapagliflozin have a therapeutic effect on diabetic atherosclerosis, which may depend on macrophages[17].

The transcription factor signal transducer and activator of transcription 3 (STAT3) is recognized to have protective effects in the heart[18]. There is growing evidence indicates that STAT3 signaling pathway can regulate the phenotype of macrophage, which STAT3 is a key factor in the polarization of M2[5, 19]. Coincidentally, Tsung-Ming Lee et al. reported that dapagliflozin induced M2 polarization through a RONS-dependent STAT3-mediated pathway in the infarcted rat heart[20]. Furthermore, cardiac-specific STAT3 knockout mice with showed a long-term decline in cardiac function after virus infection, which is associated with myocardial fibrosis[21].

These suggested that dapagliflozin plays an important role in inflammatory disease. However, the role of dapagliflozin in CVB3 induced viral myocarditis has not been reported. In view of this, we would like to explore the role of dapagliflozin in VMC induced by CVB3 and the specific molecular biological mechanism.

\section{Methods}

\subsection{Animals and virus}

Six-week-old male BALB/c mice were purchased from Vital River Laboratory Animal Technology (Beijing, China). All animals were fed in the Experimental Animal Center of Shandong Provincial Hospital (Shandong, P.R. China). All animal experiments and procedures were approved by the Ethics Committee of Shandong Provincial Hospital, and the experiments were conducted in accordance with the Guide for the Care and Use of Laboratory Animals, 8th edition, published by the National Institutes of Health (NIH Publication No. 85-23, revised 1996)[22]. The CVB3 (Nancy strain) were prepared by passage through HeLa cell cultures. Virus titers were determined through a 50\% tissue culture infectious dose (TCID50) assay of HeLa cells and calculated by the Reed-Muench method[23].

\subsection{Experiment (in vivo)}

Forty-eight male mice (18-20 g, 6 weeks old) were randomly assigned into 4 groups with 12 mice per group: Control, Dapagliflozin (a specific SGLT2 inhibitor, Bristol-Myers Squibb), VMC and the combination of VMC + Dapagliflozin. Mice were injected with $100 \mu \mathrm{l}$ of phosphate-buffered saline (PBS) containing 103 TCID50 of CVB3 intraperitoneally at day 0 to establish a VMC model as previously described in the literature, while control mice were injected with $100 \mu \mathrm{l}$ of PBS. Dapagliflozin $(0.1 \mathrm{mg} / \mathrm{kg}$ per day) dissolved in $60 \%$ propylene glycol or control agent (60\% propylene glycol) was given daily by gavage from day 1 . At day 8 , all the mice underwent echocardiography and were then executed, heart tissues and serum were taken for the next analysis. 


\subsection{Cell culture and treatment protocol}

The Raw264.7 macrophage cell line was cultured in RPMI-1640 (Life Technologies, Carlsbad, CA, USA) supplemented with $10 \%$ fetal bovine serum (FBS) (Life Technologies). $1 \times 10^{6}$ cells/well were seeded in 6well plate and incubated in serum free medium overnight before treatment. To examine the effect of dapagliflozin, Lipopolysaccharides (LPS) (Sigma) was administered to stimulate M1 macrophage activation, dapagliflozin $(10 \mu \mathrm{M})$ was given at the same time, after $24 \mathrm{~h}$ stimulates, the macrophages' phenotype distribution and inflammatory factors were observed.

\subsection{Mouse cardiac echocardiography}

Mouse cardiac echocardiography was operated in accordance with the manufacturers' instructions. Mice were anesthetized by $2 \%$ isoflurane (supplied by Shandong Provincial Hospital). Then the cardiac function parameters such as left ventricular internal dimensions in systole (LVID-s) and diastole (LVID-d), left ventricular fractional shortening (LVFS) and left ventricular ejection fraction (LVEF) were measured. The experiment was conducted in a double-blind fashion.

\subsection{Serological index measurement}

Serum cardiac troponin I (cTNI) activities were tested by Shandong Provincial Hospital. The levels of serum IL-1 $\beta$, IL-6, and TNF-a were determined by ELISA (eBioscience) following the manufacturer's instructions. All samples were measured in triplicate.

\subsection{Histopathological analysis}

Heart samples were fixed in $10 \%$ buffered formalin solution and then embedded in paraffin. The samples were sectioned ( $5 \mu \mathrm{m}$ thick) and then stained with hematoxylin and eosin (Servicebio,Wuhan,China) according to the manufacturing instructions, and the degree of inflammation was determined under 200xmagnification light microscopy. Myocardial histopathologic findings were quantified and scored for severity as follows: $0=$ no inflammation, $1=$ one to five distinct mononuclear inflammatory foci with $5 \%$ involvement or less of the cross-sectional area, $2=$ more than five distinct mononuclear inflammatory foci or over $5 \%$ but not exceeding $20 \%$ involvement of the cross-sectional area, $3=$ diffuse mononuclear inflammation involving over $20 \%$ of the area without necrosis, and $4=$ diffuse inflammation with necrosis. The analysis was performed in a double-blinded manner by a trained pathologist. Besides, myocardial fibrosis was determined by Masson staining (Servicebio,Wuhan,China) according to the manufacturing instructions. Myocardial cells were stained red and collagenous fibers were stained blue. Collagen area fraction (collagen area/field area× $100 \%$ ) was calculated by the Image-Pro Plus analysis system.

\subsection{Immunofluorescence}

Heart samples were fixed in $10 \%$ buffered formalin solution and then embedded in paraffin. The samples were sectioned ( $5 \mu \mathrm{m}$ thick). Incubate sections in 2 changes of xylene, 15 min each. Dehydrate in 2 changes of pure ethanol for 5 min, followed by dehydrate in gradient ethanol of $85 \%$ and $75 \%$ ethanol, 
respectively, 5 min each. Wash in distilled water. Immerse the slides in EDTA antigen retrieval buffer (pH 8.0) and maintain at a sub-boiling temperature for $8 \mathrm{~min}$, standing for $8 \mathrm{~min}$ and then followed by another sub-boiling temperature for 7 min. Let air cooling. Wash three times with PBS (pH 7.4) in a Rocker device, 5 min each. Block endogenous peroxidase: wash three times with PBS (pH 7.4) in a Rocker device, 5 min each eliminate obvious liquid, mark the objective tissue with liquid blocker pen. Immerse in $3 \%$ $\mathrm{H} 2 \mathrm{O} 2$ and incubate at room temperature for $25 \mathrm{~min}$, kept in dark place. Then wash again three times with PBS (pH 7.4) in a Rocker device, 5 min each. Eliminate obvious liquid, mark the objective tissue with liquid blocker pen. Cover objective tissues with $10 \%$ donkey serum at room temperature for 30 min. Incubate slides with CD68 antibody (diluted at 1:200; Servicebio) overnight at $4{ }^{\circ} \mathrm{C}$, wash slides three times with PBS ( $\mathrm{pH}$ 7.4) in a Rocker device, 5 min each. Cover objective tissue with Cy3 conjugated Goat Anti-Rabbit IgG (diluted at 1:200; Servicebio), incubate at room temperature for 50 min in dark condition. Wash slides three times with PBS ( $\mathrm{pH}$ 7.4) in a Rocker device, 5 min each. Incubate slides with TSA-FITC solution for $10 \mathrm{~min}$ in dark condition. After that, wash slides three times with TBST in a Rocker device, 5 min each. Immerse the slides in EDTA antigen retrieval buffer $(\mathrm{pH} \mathrm{8.0)}$ and maintain at a sub-boiling temperature for $8 \mathrm{~min}$, standing for $8 \mathrm{~min}$ and then followed by another sub-boiling temperature for $7 \mathrm{~min}$, to remove the antibodies combined with tissue. Incubate slides with CD163 antibody (diluted at 1:1000; Servicebio) overnight at $4{ }^{\circ} \mathrm{C}$, placed in a wet box containing a little water. Wash slides three times with PBS ( $\mathrm{pH}$ 7.4) in a Rocker device, $5 \mathrm{~min}$ each. Then throw away liquid slightly. Cover objective tissue with Cy3 conjugated Goat Anti-Rabbit IgG (diluted at 1:200; Servicebio), incubate at room temperature for 50 min in dark condition. Eliminate obvious liquid, incubate slides with spontaneous fluorescence quenching reagent for $5 \mathrm{~min}$, then wash slides under flowing water for $10 \mathrm{~min}$. Incubate with DAPI solution at room temperature for $10 \mathrm{~min}$, kept in dark place. Wash three times with PBS (pH 7.4) in a Rocker device, 5 min each. Throw away liquid slightly, then coverslip with anti-fade mounting medium. Microscopy detection and collect images by Fluorescent Microscopy. DAPI glows blue by UV excitation wavelength 330-380 $\mathrm{nm}$ and emission wavelength $420 \mathrm{~nm}$; FITC glows green by excitation wavelength 465-495 nm and emission wavelength $515-555 \mathrm{~nm}$; CY3 glows red by excitation wavelength $510-560 \mathrm{~nm}$ and emission wavelength $590 \mathrm{~nm}$.

\subsection{Quantitative real-time PCR}

Total RNA samples of myocardial infiltrating macrophages and cultured RAW264.7 were extracted with TRIzol reagent (Takara, China) in accordance with the manufacturer's instruction. First-strand complementary DNA was synthesized using $1 \mu \mathrm{g}$ of total RNA in a $20 \mu \mathrm{L}$ reaction buffer containing MMLV-RT and oligo (dT) primers (Takara, China). The mixture was incubated at $42^{\circ} \mathrm{C}$ for $60 \mathrm{~min}, 70^{\circ} \mathrm{C}$ for $15 \mathrm{~min}$, and then cooled to $4^{\circ} \mathrm{C}$. To detect the expression of genes (TNF-a, iNOS, CD206, Arginase- 1 and Stat3), cDNA was amplified with specific real-time PCR primers by using SYBR green real-time PCR kits (Takara, China). The following mRNA primer sequences were used: 


\begin{tabular}{|c|c|c|}
\hline Gene & Forward primer & Reverse primer \\
\hline TNF-a & 5'-TGTGCTCAGAGCTTTCAACAA-3' & 5'-CTTGATGGTGGTGCATGAGA-3' \\
\hline IL-1 $\beta$ & 5'-GCAACTGTTCCTGAACT-3' & 5'-ATCTTTTGGGGTCCGTCAACT-3' \\
\hline iNOS & 5'-CGAAACGCTTCACTTCCAA-3' & 5'-TGAGCCTATATGCTGTGGCT-3' \\
\hline CD206 & 5'-ACGAGCAGGTGCAGTTTACA-3' & 5'-ACATCCCATAAGCCACCTGC-3' \\
\hline Stat3 & 5'-ACGAAAGTCAGGTTGCTGCT-3' & 5'-GCTGCCGTTGTTAGACTCCT-3' \\
\hline$\beta$-actin & 5'-TGTTACCAACTGGGACGACA-3' & 5'-CTGGGTCATCTTTTCACGGT-3' \\
\hline
\end{tabular}

The quantified data were analyzed using the 2- $\Delta \Delta \mathrm{Ct}$ method[24].

\subsection{Western blot analysis}

Fresh myocardial tissue was lysed by RIPA lysis buffer (Nanjing, Nanjing, China) and proteins were extracted. Proteins were separated by SDS-PAGE and transferred to PVDF membranes. The membrane was closed with $5 \%$ bovine serum albumin and then incubated with the appropriate primary antibody overnight at $4^{\circ} \mathrm{C}$, followed by washing with TBS-Tween and incubation with the appropriate secondary antibody at room temperature for $1 \mathrm{~h}$. Spots were detected using an ECL system (amersham, UK) and optical density was measured using ImageJ software. The following antibodies were used: iNOS (Cell Signaling Technology, 13120S), CD206 (Abcam, ab125028), (Abcam, ab28946), IL-6(Cell Signaling Technology, 12912S), Stat3(Cell Signaling Technology, 9139S), Phospho-Stat3(Cell Signaling Technology, 9145S), GAPDH polyclonal antibody (Proteintech, No.10494-1-AP), and $\beta$-tubulin polyclonal antibody (Proteintech, No.10094-1-AP).

\subsection{Statistical analysis}

Survival rate was analyzed using the Log-rank test. Comparisons between two groups were performed with Unpaired t-test. For comparisons of three groups, One-way ANOVA analysis was used, followed by post-test using Tukey multiple comparison test. Correlations were determined by Pearson's correlation coefficients. All statistical analyses were performed using the commercially available software SPSS version 20.0, GraphPad Prism version 7. The data are presented as the mean \pm SD in the figures, with $\mathrm{P}<0.05$ considered significant.

\section{Results}

\section{General manifestation changes}

As expected, the symptoms of viral myocarditis in the virus group were evident, including a rough grey color, reduced activity and diet, and dyspnea at rest. These signs were improved to varying degrees in the dapagliflozin group after dapagliflozin treatment. We also monitored weight loss, the bodyweight of the virus group decreased continuously from day 2 post-infection compared to the control group, and 
dapagliflozin have no effect on this change (Fig. 1a). The survival rate in the virus group was significantly lower than in the control group, while dapagliflozin-treated mice showed significant improvement (Fig. 1b). Taken together, dapagliflozin application significantly prevented the progression of CVB3induced general presentation and improved survival.

\section{Dapagliflozin attenuates the myocardial dysfunction}

We further assayed the potential of dapagliflozin to inhibit CVB3-mediated cardiac dysfunction by echocardiography on day 8. As Table 1 depicted, CVB3 infection led to significant reduced cardiac contractility and impaired diastolic function, while dapagliflozin administration could reverse cardiac function decline. Thus, these data support the notion that dapagliflozin participates in CVB3 induced myocardial dysfunction in vivo.

Table 1

\begin{tabular}{|c|c|c|c|c|}
\hline & Control & Dapa & CVB & CVB+Dapa \\
\hline LVID-(Mm) & $2.66 \pm 0.25$ & $2.65 \pm 0.31$ & $3.88 \pm 0.21^{\star \star \star}$ & $3.36 \pm 0.20^{\# \# \#}$ \\
\hline LVID-s(Mm) & $1.91 \pm 0.37$ & $1.90 \pm 0.21$ & $2.80 \pm 0.15^{\star \star \star}$ & $2.49 \pm 0.06 \# \#$ \\
\hline LVEF(\%) & $74.17 \pm 6.26$ & $74.88 \pm 5.53$ & $39.76 \pm 6.57^{\star \star \star \star}$ & $58.79 \pm 6.08 \# \# \#$ \\
\hline LVFS(\%) & $43.98 \pm 2.85$ & $44.82 \pm 3.54$ & $24.27 \pm 1.67^{\star \star \star \star}$ & $35.93 \pm 1.87 \# \# \#$ \\
\hline \multicolumn{5}{|c|}{ 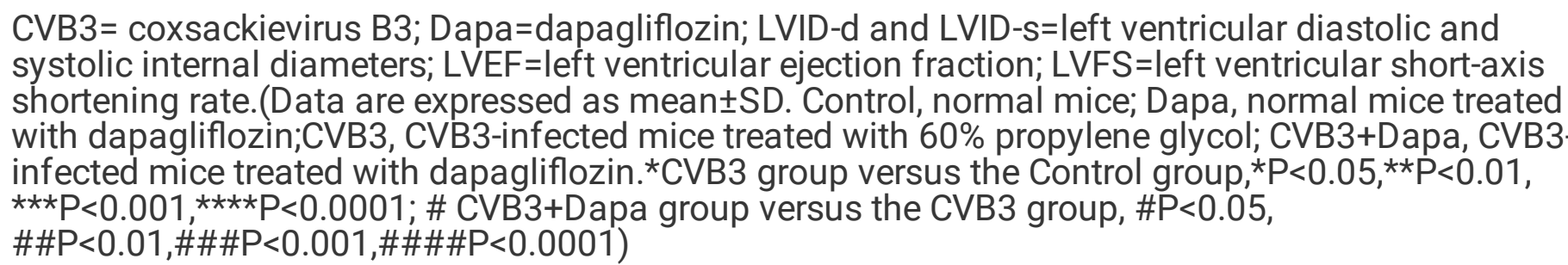 } \\
\hline
\end{tabular}

\section{Inhibitory effects of dapagliflozin on myocarditis-affected inflammation}

Next, we examined the protective role of dapagliflozin against myocarditis-affected inflammation and cardiac remodeling in vivo on day 8 . As shown in Fig. 2a, virus myocarditis elicited typical cardiac histopathological changes in the myocardium. Severe inflammatory lesions infiltrated by large numbers of inflammatory cells were observed, while dapagliflozin treatment significantly reduced the infiltration of inflammatory cells. Further quantitative evaluation revealed a significant reduction of mean pathological myocarditis scores in the dapagliflozin group compared with the virus group during the acute phase of virus myocarditis $(P<0.0001$, Fig. $2 b)$. Consistent with these findings, the serum $c T n l$ activities were also reduced in the mice treated with dapagliflozin $(P<0.05$, Fig. $2 \mathrm{c})$. For evaluation of cardiac remodeling, heart sections were stained with Masson trichrome dye to visualize interstitial collagen content. As shown in Fig. 2d, CVB3 resulted in a visible fibrosis in interstitial tissue of myocardium in the Virus group. Quantification of fibrosis (Fig. 2e) also showed a statistical increase in collagen deposition of Virus mice. 
Contrary to this, in dapagliflozin treatment mice, the collagen area fraction was significantly lower than that in the Virus group ( $P<0.0001$, Fig. $2 d, e)$. Taken together our data indicate that dapagliflozin protected mice against the pathological changes of viral myocarditis.

\section{Percentages of M2 are increased and M1 are decreased in dapagliflozin treatment mice}

The phenotype of macrophage is directly regulated by the composition of the microenvironment, including cytokine profiles. We therefore examined the levels of inflammatory or anti-inflammatory cytokines in each group. Plasma samples were collected at day 8 and analyzed by ELISA. The levels of IL-1 $\beta$, IL- 6 and TNF- $a$, cytokines related to macrophage, were higher in the Virus group than in the Control group, but were lower in dapagliflozin-treated mice $(P<0.05$, Fig. 3a, b, c). To identify the subtype of infiltrated macrophages in myocardium, the marker for M1 (CD68+) and M2 (CD163+) was examined (Fig. 3d). Immunofluorescence showed that there were a large number of CD68+ macrophages infiltrated in the CVB groups, and CD163+ macrophages were more frequent in the dapagliflozin. These data suggest dapagliflozin reduces M1 macrophage differentiation while increasing M2. Besides, the mRNA expression of iNOS were significantly upregulated in the myocardial tissue of mice in the CVB3 group compared with the control group ( $P<0.05$, Fig. $4 a$ ), which was significantly downregulated in the dapagliflozin-treated group compared with the CVB3 group ( $P<0.05$, Fig. 4a). In addition, we found that the mRNA expression of CD206 was significantly decreased in VMC mice, and dapagliflozin treatment alleviated this change $(P<0.05$, Fig. $4 b)$. These results suggest that dapagliflozin decreased the percentages of M1 and increased M2 in VMC mice.

\section{Dapagliflozin activates the Stat3 signaling pathway in VMC}

Previous studies have demonstrated that Stat 3 is important in regulating the phenotype of macrophage, here we investigate the effect of dapagliflozin on Stat3 in VMC. We found that Stat3 was significantly downregulated in the myocardial tissue of mice in the CVB3 group compared with the control group, while it was significantly upregulated in the dapagliflozin-treated group compared with the CVB3 group

(Fig. 4c). Consistent with this, IL-6 was significantly upregulated in the myocardial tissue of mice in the CVB3 group compared with the control group, while it was significantly downregulated in the dapagliflozin-treated group compared with the CVB3 group (Fig. 3b,4c).

\section{Discussion}

Viral myocarditis is considered as an inflammatory disease and immunomodulatory therapy has drawn intensive attention. The existing literature suggests that macrophages play a crucial role in CVB3-induced myocarditis[25]. In this study, we focused on the therapeutic potential of dapagliflozin for VMC. Results showed that dapagliflozin treatment inhibited macrophage polarizing to M1 type, thereby alleviating the severity of VMC. In addition, dapagliflozin inhibited inflammatory cell infiltration and pro-inflammatory cytokine production in VMC mice infected with CVB3. These results indicate that dapagliflozin has the potential to be an effective drug for the treatment of VMC. 
The pathogenic mechanism of the VMC includes two sequential processes. In the early stage, the virus enters the cardiac muscle cells by receptor-mediated endocytosis. During late-stage infection, large numbers of immune cells accumulate in the infected heart tissue and strongly augment the expression of proinflammatory cytokines, resulting in massive inflammation and aggravated heart injury[26]. Therefore, immunosuppression is the focus of treatment research at present and many drugs have been reported have a protective effect on VMC through anti-inflammatory effects[27]. The existing literature indicates that macrophages play a crucial role in CVB3-induced myocarditis, in particular, differential-phenotype macrophages may generate an opposite inflammatory response. Consistent with this, some drugs work by promoting the polarization of macrophages towards M2[28].

Dapagliflozin have been reported to have an anti-inflammatory function and can influence the phenotype of macrophage[17]. It has been reported that SGLT2 inhibition attenuates inflammation, including levels of CRP, IL-6, and TNF-a, and the progression of diabetic nephropathy[29-31]. A recent study suggested that empagliflozin attenuates NIrp3 inflammasome activation in the kidney and liver of male C57BL/6 mice fed high-fat-high-sugar diet[32]. In this study, dapagliflozin treatment improved the survival rate and left ventricular function, as well as decreased level of serum cTnl and myocardial inflammation in VMC. We observed reduced expression of iNOS (marker of M1), as well as elevated expression of CD206(marker of M2), suggesting that dapagliflozin may protect VMC by inhibiting macrophage polarizing to $\mathrm{M} 1$.

The transcription factor signal transducer and activator of transcription 3 is an important mediator of the inflammatory process, known as a transcription activator of IL-6. Diana Lindner et al. found that the cardiac function in STAT3-KO mice was significantly decreased in contrast to the infected WT mice, revealed a protective function of STAT3 expressed in cardiomyocytes after CVB3-induced myocarditis. Besides, in other cardiac damages such as myocardial infarction or doxorubicin-induced cardiomyopathy, STAT3 in cardiomyocytes prevents uncontrolled fibrosis and clinical progression to DCM. Therefore, STAT3 seems to be a crucial factor for the resolution of viral myocarditis. We found that dapagliflozin treatment increased anti-inflammatory macrophage polarization and reduced cardiac injury following VMC via activating Stat3 signal pathway. However, this finding still requires further exploration due to the incompletely validated correlation between dapagliflozin and M1/M2 phenotypes seen in the current study.

Nevertheless, we still could not exclude the function of other type cells which may be involved in the viral myocarditis. RT-PCR confirmed that SGLT2 and SGLT1 are not expressed in RAW264.7 and the hearts of the Balb/c mice (data not shown), which means the anti-inflammatory, anti-fibrosis effects are likely SGLT2-independent. And this dose of dapagliflozin has been shown that blood glucose levels would not be different from those in the control group, thus enabling us to assess direct drug effects independently from blood glucose control. Although some experts tried to link the favorable effects on clinical outcomes solely to the diuretic effects of the drugs, others expressed doubts and suggested that other mechanisms may exist. 
In summary, we showed for the first time that dapagliflozin can alleviate CVB3-induced myocardial inflammation. Therefore, our findings suggest a therapeutic potential of dapagliflozin in the treatment of viral myocarditis.

\section{Conclusion}

In the mouse model of CVB3-induced viral myocarditis, dapagliflozin inhibits the production and release of inflammatory cytokines IL-1 $\beta$,IL- 6 and TNF- $\alpha$, inhibiting macrophage polarization toward M1-type, reduced inflammatory infiltration, and significantly improved the survival rate of mice, these effects may through Stat3 signal pathway.

\section{Declarations}

\section{Funding and Acknowledgements:}

This work was supported by the National Natural Science Foundation of China (No.81770382); Natural Science Foundation of Shandong Province (No. ZR2020MH031); Shandong Provincial Key R\&D Program (NO. 2018GSF118109); Shandong Province Medicine and Health Science Technology Development Project (NO. 2018WS272); Shandong Province Postdoctoral Innovation Fund (NO. 201903087); Jinan Clinical Medicine Science and Technology Innovation Plan (NO. 201907033).

\section{Competing Interests:}

The authors have no relevant financial or non-financial interests to disclose..

\section{Author Contributions:}

All authors contributed to the study conception and design. Material preparation, data collection and analysis were performed by [Runfa Zhou], [Xiaoning Song] and [Joanne Tran]. The first draft of the manuscript was written by [Pengcheng Yan] and all authors commented on previous versions of the manuscript. All authors read and approved the final manuscript.

\section{Ethics approval:}

This study was performed in line with the principles of the Declaration of Helsinki. Approval was granted by the Ethics Committee of Shandong Provincial hospital (Date2020/SDU.SPHEARC-A099).

\section{References}

1. Fung G, Luo H, Qiu Y, Yang D, McManus B: Myocarditis. Circ Res 2016, 118(3):496-514.

2. Kindermann I, Barth C, Mahfoud F, Ukena C, Lenski M, Yilmaz A, Klingel K, Kandolf R, Sechtem U, Cooper LT et al: Update on myocarditis. J Am Coll Cardiol 2012, 59(9):779-792. 
3. Corsten MF, Schroen B, Heymans S: Inflammation in viral myocarditis: friend or foe? Trends in molecular medicine 2012, 18(7):426-437.

4. Mantovani A, Sica A, Sozzani S, Allavena P, Vecchi A, Locati M: The chemokine system in diverse forms of macrophage activation and polarization. Trends in immunology 2004, 25(12):677-686.

5. Gordon S, Martinez FO: Alternative activation of macrophages: mechanism and functions. Immunity 2010, 32(5):593-604.

6. Li K, Xu W, Guo Q, Jiang Z, Wang P, Yue Y, Xiong S: Differential macrophage polarization in male and female BALB/c mice infected with coxsackievirus B3 defines susceptibility to viral myocarditis. Circ Res 2009, 105(4):353-364.

7. Yan Zhang 1 SC, Xiaoxue Ding 3, Can Lu 3, Ruodan Wu 3, Haiyan Wu 3, Yiyi 7 Shang 4, Mingjie Pang 3 , *: microRNA-30a-5p silencing polarizes macrophages towards M2 2 phenotype to alleviate cardiac injury following viral myocarditis by 3 targeting SOCS1.

8. Wang C, Dong C, Xiong S: IL-33 enhances macrophage M2 polarization and protects mice from CVB3-induced viral myocarditis. J Mol Cell Cardiol 2017, 103:22-30.

9. Zannad F, Ferreira JP, Pocock SJ, Anker SD, Butler J, Filippatos G, Brueckmann M, Ofstad AP, Pfarr E, Jamal $W$ et al: SGLT2 inhibitors in patients with heart failure with reduced ejection fraction: a metaanalysis of the EMPEROR-Reduced and DAPA-HF trials. The Lancet 2020, 396(10254):819-829.

10. Verma S, Mazer CD, Fitchett D, Inzucchi SE, Pfarr E, George JT, Zinman B: Empagliflozin reduces cardiovascular events, mortality and renal events in participants with type 2 diabetes after coronary artery bypass graft surgery: subanalysis of the EMPA-REG OUTCOME(R) randomised trial. Diabetologia 2018, 61(8):1712-1723.

11. McMurray JJV, DeMets DL, Inzucchi SE, Køber L, Kosiborod MN, Langkilde AM, Martinez FA, Bengtsson O, Ponikowski P, Sabatine MS et al: A trial to evaluate the effect of the sodium-glucose co-transporter 2 inhibitor dapagliflozin on morbidity and mortality in patients with heart failure and reduced left ventricular ejection fraction (DAPA-HF). European Journal of Heart Failure 2019, 21(5):665-675.

12. Uthman L, Kuschma M, Romer G, Boomsma M, Kessler J, Hermanides J, Hollmann MW, Preckel B, Zuurbier CJ, Weber NC: Novel Anti-inflammatory Effects of Canagliflozin Involving Hexokinase II in Lipopolysaccharide-Stimulated Human Coronary Artery Endothelial Cells. Cardiovasc Drugs Ther 2020.

13. EIMahdy MK, Helal MG, Ebrahim TM: Potential anti-inflammatory effect of dapagliflozin in HCHF diet- induced fatty liver degeneration through inhibition of TNF-alpha, IL-1beta, and IL-18 in rat liver. Int Immunopharmacol 2020, 86:106730.

14. Li C, Zhang J, Xue M, Li X, Han F, Liu X, Xu L, Lu Y, Cheng Y, Li T et al: SGLT2 inhibition with empagliflozin attenuates myocardial oxidative stress and fibrosis in diabetic mice heart. Cardiovasc Diabetol 2019, 18(1):15.

15. Tang L, Wu Y, Tian M, Sjostrom CD, Johansson U, Peng XR, Smith DM, Huang Y: Dapagliflozin slows the progression of the renal and liver fibrosis associated with type 2 diabetes. Am J Physiol 
Endocrinol Metab 2017, 313(5):E563-E576.

16. Yagihashi S, Terami N, Ogawa D, Tachibana H, Hatanaka T, Wada J, Nakatsuka A, Eguchi J, Horiguchi CS, Nishii N et al: Long-Term Treatment with the Sodium Glucose Cotransporter 2 Inhibitor, Dapagliflozin, Ameliorates Glucose Homeostasis and Diabetic Nephropathy in db/db Mice. PLoS ONE 2014, 9(6).

17. Leng W, Ouyang X, Lei X, Wu M, Chen L, Wu Q, Deng W, Liang Z: The SGLT-2 Inhibitor Dapagliflozin Has a Therapeutic Effect on Atherosclerosis in Diabetic ApoE(-/-) Mice. Mediators Inflamm 2016, 2016:6305735.

18. Kurdi M, Zgheib C, Booz GW: Recent Developments on the Crosstalk Between STAT3 and Inflammation in Heart Function and Disease. Front Immunol 2018, 9:3029.

19. Ma Y, Mouton AJ, Lindsey ML: Cardiac macrophage biology in the steady-state heart, the aging heart, and following myocardial infarction. Trans/ Res 2018, 191:15-28.

20. Lee TM, Chang NC, Lin SZ: Dapagliflozin, a selective SGLT2 Inhibitor, attenuated cardiac fibrosis by regulating the macrophage polarization via STAT3 signaling in infarcted rat hearts. Free Radic Biol Med 2017, 104:298-310.

21. Lindner D, Hilbrandt M, Marggraf K, Becher PM, Hilfiker-Kleiner D, Klingel K, Pauschinger M, Schultheiss HP, Tschope C, Westermann D: Protective Function of STAT3 in CVB3-Induced Myocarditis. Cardiol Res Pract 2012, 2012:437623.

22. <Animals, Guide for the Care and Use of Laboratory Animals..pdf>.

23. Henke A HS, Stelzner A, Whitton JL.: The role of CD8+ T lymphocytes in coxsackievirus B3-induced myocarditis. Journal of virology 1995.

24. Livak KJ, Schmittgen TD: Analysis of relative gene expression data using real-time quantitative PCR and the 2(-Delta Delta C(T)) Method. Methods 2001, 25(4):402-408.

25. Caforio AL, Pankuweit S, Arbustini E, Basso C, Gimeno-Blanes J, Felix SB, Fu M, Helio T, Heymans S, Jahns R et al: Current state of knowledge on aetiology, diagnosis, management, and therapy of myocarditis: a position statement of the European Society of Cardiology Working Group on Myocardial and Pericardial Diseases. Eur Heart J 2013, 34(33):2636-2648, 2648a-2648d.

26. Esfandiarei M, McManus BM: Molecular biology and pathogenesis of viral myocarditis. Annu Rev Pathol 2008, 3:127-155.

27. Li L, Li L, Xiao L, Shangguan J: Progranulin ameliorates coxsackievirus-B3-induced viral myocarditis by downregulating Th1 and Th17 cells. Exp Cell Res 2018, 367(2):241-250.

28. Gao S, Zhou J, Liu N, Wang L, Gao Q, Wu Y, Zhao Q, Liu P, Wang S, Liu Y et al: Curcumin induces M2 macrophage polarization by secretion IL-4 and/or IL-13. J Mol Cell Cardiol 2015, 85:131-139.

29. Tahara A, Kurosaki E, Yokono M, Yamajuku D, Kihara R, Hayashizaki Y, Takasu T, Imamura M, Li Q, Tomiyama $\mathrm{H}$ et al: Effects of SGLT2 selective inhibitor ipragliflozin on hyperglycemia, hyperlipidemia, hepatic steatosis, oxidative stress, inflammation, and obesity in type 2 diabetic mice. Eur $J$ Pharmacol 2013, 715(1-3):246-255. 
30. Tahara A, Kurosaki E, Yokono M, Yamajuku D, Kihara R, Hayashizaki Y, Takasu T, Imamura M, Li Q, Tomiyama $\mathrm{H}$ et al: Effects of sodium-glucose cotransporter 2 selective inhibitor ipragliflozin on hyperglycaemia, oxidative stress, inflammation and liver injury in streptozotocin-induced type 1 diabetic rats. J Pharm Pharmacol 2014, 66(7):975-987.

31. De Nicola L, Gabbai FB, Liberti ME, Sagliocca A, Conte G, Minutolo R: Sodium/Glucose Cotransporter 2 Inhibitors and Prevention of Diabetic Nephropathy: Targeting the Renal Tubule in Diabetes. American Journal of Kidney Diseases 2014, 64(1):16-24.

32. Benetti E, Mastrocola R, Vitarelli G, Cutrin JC, Nigro D, Chiazza F, Mayoux E, Collino M, Fantozzi R: Empagliflozin Protects against Diet-Induced NLRP-3 Inflammasome Activation and Lipid Accumulation. J Pharmacol Exp Ther 2016, 359(1):45-53.

\section{Figures}



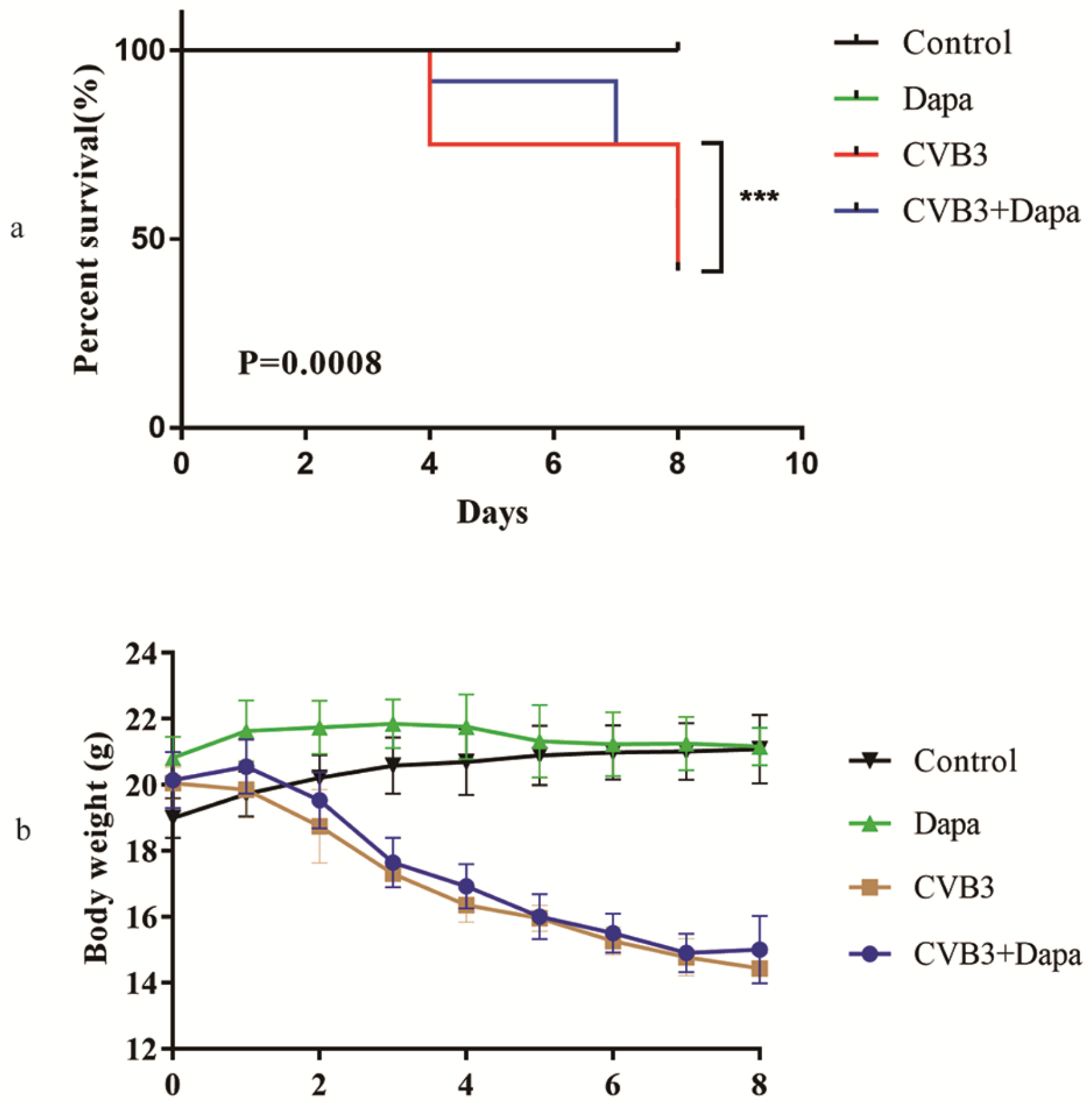

Days post CVB3 infection

Figure 1

Dapagliflozin (Dapa) treatment ameliorates the general manifestations of myocarditis. Viral myocarditis model was created in BALB/C mice as described in Methods. Monitor the (a)body weight change and (b) survival rate of mice from day 0 to day8. Data are shown as mean \pm SD. (Control, normal mice; Dapa, normal mice treated with dapagliflozin; CVB3, CVB3-infected mice treated with $60 \%$ propylene glycol; 
CVB3+Dapa, CVB3-infected mice treated with dapagliflozin. *CVB3 +Dapa group versus CVB3 group, $\left.{ }^{\star} \mathrm{P}<0.05,{ }^{\star *} \mathrm{P}<0.01,{ }^{\star * \star} \mathrm{P}<0.001, * \star \star \star P<0.0001\right)$

\section{Figure 2}

Protective effects of dapagliflozin in CVB3-induced viral myocarditis. (a) Hematoxylin-eosin staining to observe the inflammatory response to myocarditis. Red stained area shows myocardial tissue, blue staining shows inflammatory cell infiltration (magnification: $\times 200$, Scale bar: $100 \mu \mathrm{m}$ ). (b)The severity of myocarditis was scored using standard 0-4 grading scale. Data are expressed as mean $\pm S D$.(c)Serum myocardial injury markers cardiac troponin I (CTnl) were measured by ELISA. (d) Masson staining to observe the inflammatory response to myocarditis. Myocardial cells were stained red and collagenous fibers were stained blue (magnification: $\times 200$, Scale bar: $100 \mu \mathrm{m}$ ). (e) Collagen area fraction (collagen area/field area $\times 100 \%$ ) was calculated by the Image-Pro Plus analysis system. Data were expressed as mean $\pm S D$. (Control, normal mice; Dapa, normal mice treated with dapagliflozin; CVB3, CVB3-infected mice treated with $60 \%$ propylene glycol; CVB3+Dapa, CVB3-infected mice treated with dapagliflozin. *CVB3 group versus the Control group, ${ }^{*}<<0.05,{ }^{\star *} P<0.01$, ${ }^{* \star *} P<0.001$, ${ }^{\star \star \star *} P<0.0001$; \# CVB3+Dapa group versus

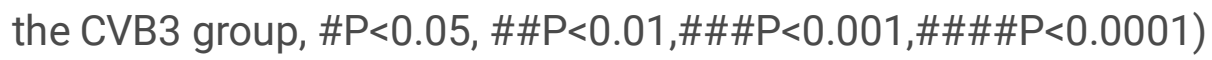

\section{Figure 3}

Dapagliflozin treatment reduces proinflammatory cytokine production and M1. (a, b, c) Quantification of IL-1 $\beta$, IL- 6 and TNF- $\alpha$ levels in myocardial tissue by Elisa analysis. (d) Immunofluorescent double staining of myocardial tissue with antibodies to CD68 and CD163. Red indicates CD68(the marker for M1), green CD163(the marker for M2), and blue DAPI-stained cellular nuclei. Data were expressed as mean \pm SD. (Control, normal mice; Dapa, normal mice treated with dapagliflozin; CVB3, CVB3-infected mice treated with $60 \%$ propylene glycol; CVB3+Dapa, CVB3-infected mice treated with dapagliflozin. *CVB3 group versus the Control group, ${ }^{*} P<0.05,{ }^{\star \star} P<0.01,{ }^{\star \star \star *} P<0.001, \star \star \star \star * P<0.0001$; \# CVB3+Dapa group versus the CVB3 group, $\# \mathrm{P}<0.05, \# \# \mathrm{P}<0.01, \# \# \# \mathrm{P}<0.001, \# \# \# \# \mathrm{P}<0.0001)$

\section{Figure 4}

Dapagliflozin inhibits macrophage polarizing toward M1-type and activates the Stat3 pathway in viral myocarditis. ( $a, b)$ Quantitative reverse transcription polymerase chain reaction (qRT-PCR) was used to detect iNOS, CD206 mRNA levels. (c)Western blot detection of iNOS, IL-6 and Stat3 expression. Data were expressed as mean $\pm S D$. Data were expressed as mean $\pm S D$. All the experiments were repeated at least three times. (Control, normal mice; Dapa, normal mice treated with dapagliflozin;CVB3,CVB3-infected 
mice treated with $60 \%$ propylene glycol; CVB3+Dapa, CVB3-infected mice treated with dapagliflozin. ${ }^{\star} C V B 3$ group versus the Control group, ${ }^{*} P<0.05$, ${ }^{\star \star} P<0.01,{ }^{* \star \star} P<0.001$, ${ }^{\star \star * \star} P<0.0001$; \#

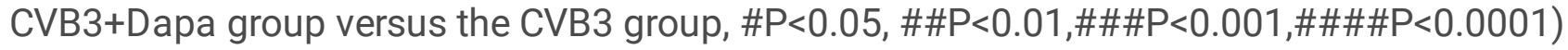

